\title{
LEVANTAMENTO QUALITATIVO E REPRODUTIVO DA ORNITOFAUNA DOS PARQUES TARQUÍNIO JOSLIN DOS SANTOS E PARQUE ECOLÓGICO PAULO GORSKI, CASCAVEL - PR
}

Kathleen Jeniffer Model ${ }^{1}$

Marcelo Bevilacqua Remor ${ }^{2}$

Jéssica Engel do Nascimento ${ }^{3}$

MODEL, K. J.; REMOR, M. B.; NASCIMENTO, J. E. do. Levantamento qualitativo e reprodutivo da ornitofauna dos parques Tarquínio Joslin dos Santos e parque ecológico Paulo Gorski, Cascavel - PR. Arq. Ciênc. Vet. Zool. UNIPAR, Umuarama, v. 17, n. 2, p. 107-114, abr./jun. 2014.

RESUMO: O presente estudo tem como objetivo o levantamento qualitativo e caracterização do comportamento reprodutivo da ornitofauna de hábitos diurnos no Parque Tarquínio Joslin dos Santos (P1) e no Parque Ecológico Paulo Gorski (P2), em Cascavel - PR, a fim de identificar interferências antrópicas na conservação e na diversidade nos parques urbanos. A composição da ornitofauna e o comportamento reprodutivo foram realizados por observações diretas auxiliadas com um guia de identificação de aves. Em P1 registrou-se 38 espécies, 20 famílias, destas destacam-se Basileuterus flaveolus e Sporophila plumbea que se encontram ameaçadas de extinção, e Dendroica estriata, por ser uma ave migratória. No P2 registrou-se 37 espécies pertencentes a 21 famílias. A reprodução foi analisada em dois parâmetros, nidificação e comportamento. Além disso obtidos dados morfométricos como altura em relação ao solo, topo ไbase, profundidade da câmara oológica, diâmetro maior e menor, substrato de apoio e material de construção dos ninhos, se estes ninhos se encontravam ativos quantificava-se o número de ovos e o padrão de coloração. No P1 ocorreu nidificação de Zenaida auriculata, Furnarius rufus, Pitangus sulphuratus e Machetornis rixosa, apenas da primeira foi possível coletar dados morfométricos e comportamentais. Já no P2 nidificaram Zenaida auriculata, Furnarius rufus, Machetornis rixosa, Megarhynchus pitangua, Turdus rufiventris, Crotophaga ani e Butorides striata, sendo também possível apenas a coleta de dados morfométricos em ninhos de Zenaida, desta e das demais foram registrados comportamentos de defesa do ninho e incubação de ninhegos e ovos. Ressalta-se a importância da conservação dos parques urbanos para manutenção da biodiversidade de aves local.

PALAVRAS-CHAVE: Ornitofauna. Nidificação. Morfometria. Comportamento reprodutivo.

\section{QUALITATIVE AND REPRODUCTIVE SURVEY OF ORNITHOFAUNA AT PARQUE TARQUÍNIO JOSLIN DOS SANTOS AND PARQUE ECOLÓGICO PAULO GORSKI, CASCAVEL - PR}

\begin{abstract}
This study aims to survey and qualitatively characterize the reproductive behavior of the diurnal ornithofauna at Parque Tarquínio Joslin dos Santos (P1) and Parque Ecológico Paulo Gorski (P2) in the city of Cascavel - PR, in order to identify anthropogenic interference in the conservation and diversity of urban parks. The bird composition and reproductive behavior were assessed by direct observations with the aid of a guide for identifying birds. In P1, a total of 38 species were recorded, from 20 families. Among these, it is important to highlight Basileuterus flaveolus and Sporophila plumbea, which are endangered, and Dendroica estriata, a migratory bird. In P2, a total of 37 species were recorded, belonging to 21 families. Reproduction was analyzed using two parameters - nesting and behavior. Morphometric data, such as height from the ground, top/bottom, depth of oologic chamber, with larger and smaller diameter, supporting substrate and construction material of nests. If these nests were considered active, the number and color pattern of eggs were quantified. In P1, nesting of Zenaida auriculata, Furnarius rufus, Pitangus sulphuratus and Machetornis rixosa, were found only, but it was only possible to collect morphometric and behavioral data from the first species. However, in P2, nests of Zenaida auriculata, Furnarius rufus, Machetornis rixosa, Megarhynchus pitangua, Turdus rufiventris, Crotophaga ani and Butorides striata were found, with morphometric data being collected only from Zenaida nests. From this species and the other ones, it was possible to record the behaviors of nest defense and incubation of eggs and hatchlings. It is important to emphasize the need to preserve urban parks for the maintenance of the biodiversity of local birds.
\end{abstract}

KEYWORDS: Ornithofauna. Nesting. Morphology. Reproductive behavior.

\section{ALZAMIENTO CUALITATIVO Y REPRODUCTIVO DE LA ORNITOFAUNA DE LOS PARQUES TARQUÍNIO JOSLIN DOS SANTOS Y PARQUE ECOLÓGICO PAULO GORSKI, CASCAVEL - PR}

RESUMEN: Esta investigación ha tenido como objetivo el alzamiento cualitativo y caracterización del comportamiento reproductivo de la ornitofauna de hábitos diurnos en el Parque Tarquínio Joslin dos Santos (P1) y en el Parque Ecológico

DOI: https://doi.org/10.25110/arqvet.v17i2.2014.4928

${ }^{1}$ Graduada em Ciências Biológicas Bacharelado, Universidade Estadual do Oeste do Paraná - Unioeste, Bairro Universitário, Rua Agronomia, nº 1276, CEP: 85.819-110, Cascavel, PR, Brasil. kathleen05jmodel@gmail.com;

${ }^{2}$ Universidade Estadual do Oeste do Paraná - UNIOESTE, Pós-graduação em Engenharia Agrícola. Rua Universitária n. 2069, Jardim Universitário, Cascavel, PR. CEP: 85.819-110. remor_hotmail.com;

${ }^{3}$ Graduanda de Ciências Biológicas Licenciatura, Universidade Estadual do Oeste do Paraná - Unioeste, Bairro Universitário, Rua Agronomia, $\mathrm{n}^{\circ}$ 1276, CEP 85.819-110, Cascavel, PR, Brasil. jessicaenge193@hotmail.com 
Paulo Gorski (P2), en Cascavel - PR, a fin de identificar interferencias antrópicas en la conservación y en la diversidad de bosques urbanos. La composición de la ornitofauna y el comportamiento reproductivo han sido realizados a través de observaciones directas, auxiliadas con un guía de identificación de aves. En P1 se registró 38 especies, 20 familias de estas se destacan Basileuterus flaveolus y Sporophila plúmbea que se encuentran amenazadas de extinción, y Dendroica estriata, por ser un ave migratoria. En el P2 se registró 37 especies pertenecientes a 21 familias. La reproducción ha sido analizada en dos parámetros: nidificación y comportamiento. Además de eso, obtenidos datos morfo métricos como altura en relación al suelo, topo, base, profundidad de la cámara, diámetro mayor y menor, substrato de apoyo y material de construcción de los nidos, si estos nidos se encontraban activos se cuantificaba el número de huevos y el estándar de coloración. En el P1 ocurrió nidificación de Zenaida auriculata, Furnarius rufus, Pitangus sulphuratus e Machetornis rixosa, solo de la primera ha sido posible recoger datos morfo métricos y de comportamiento. Ya en el P2 nidificaron Zenaida auriculata, Furnarius rufus, Machetornis rixosa, Megarhynchus pitangua, Turdus rufiventris, Crotophaga ani y Butorides striata, siendo posible recoger datos morfo métricos en nidos de Zenaida, de esta y de las demás han sido registrados comportamientos de defensa del nido e incubación de pichones y huevos. Se destaca la importancia de la conservación de los bosques urbanos para manutención de la biodiversidad de aves local.

PALABRAS CLAVE: Ornitofauna. Nidificación. Morfometría. Comportamiento reproductivo.

\section{Introdução}

O crescimento de centros urbanos é um dos componentes de fragmentação de habitats, isolando os remanescentes de ambiente natural, que ficam inseridos no perímetro urbano. Praças, parques e fundos de vale são exemplos de áreas verdes inseridas na malha urbana. Elas apresentam diversidade de aves, mas esta, na grande maioria, é constituída por novas colonizações, pois geralmente estes locais são reflorestados e com forte interferência humana na composição da estrutura vegetacional (LOPES; ANJOS, 2006). Estas áreas servem de abrigo, área de alimentação e reprodução de diversos grupos animais, bem como local de pouso para aves migratórias (SCHERER et al., 2006).

Os levantamentos avifaunísticos são importantes ferramentas para avaliação da integridade das áreas verdes urbanas. Estudos descrevem a importância da manutenção das áreas verdes para a conservação das espécies de aves, entre eles destacam-se Lopes e Anjos (2006); De Carvalho (2006); Conterno e Cândido(2001); Poletto (2002). Além disso, segundo Bartholomew (1986), o verdadeiro conhecimento de um organismo baseia-se no estudo de sua história natural, incluindo o conhecimento de aspectos reprodutivos das espécies. Esse tipo de informação também é essencial para a definição segura de estratégias para a conservação e manejo de espécies, e para testar hipóteses a cerca da evolução destas características (ROBINSON; ROBINSON; EDWARDS, 2000).

Um dos aspectos relevantes para que uma espécie se estabeleça em novos locais é a nidificação. As aves têm, de certo modo, exigências específicas para os locais dos ninhos, como fatores abióticos, por exemplo, o microclima, e bióticos, como a estrutura da vegetação citado por Smith (1997), a predação por Olmos (2003), nidoparasitismo por Larison et al. (1998), ectoparasitismo por Oppliger, Richner e Christe (1994), comportamento social por Rodrigues (1996) e aprendizado por Hatchwell et al. (1999).

Estudos da biologia reprodutiva são importantes ferramentas para identificar estratégias de conservação das espécies nas áreas verdes urbanas. No Estado do Paraná alguns estudos descrevem a biologia reprodutiva de algumas espécies, dentre eles vale ressaltar os trabalhos realizados por Loures-Ribeiro, Gimenes e Anjos (2003) com Ictinia plúmbea, Lopes e Anjos (2005) com comportamento de Nyctibius griséus, Costa (2002) com Vanellus chilensis e Guaraldo e
Gussoni (2006) com a nidificação de Zenaida auriculata.

Nesse contexto, o presente estudo teve como objetivo o levantamento qualitativo avifaunístico e caracterização do comportamento reprodutivo das espécies de aves de hábitos diurnos no Parque Tarquínio Joslin dos Santos e no Parque Ecológico Paulo Gorski, em Cascavel - PR, a fim de identificar interferências antrópicas na conservação e na diversidade nos parques urbanos.

\section{Material e Métodos}

\section{Área de Estudo}

\section{Parque Tarquínio Joslin dos Santos (P1):}

Parque municipal batizado em homenagem ao primeiro farmacêutico da cidade, construído no bosque municipal do Parque São Paulo, localizado na Rua Carlos de Carvalho, esquina com Hyeda Baggio Mayer (24⒌'19'S $53^{\circ} 27^{\prime} 41^{\prime \prime} \mathrm{W}$ ) (Figura 1). Constituído por meio do decreto municipal $3402 / 92$, o parque possui $17.600 \mathrm{~m}^{2}$ e é bastante utilizado pela população como área de lazer. É composto de vegetação caracterizado como floresta ombrófila mista, embora em muitos pontos tenha sido realizado plantio de espécies exóticas.

\section{Parque Ecológico Paulo Gorski (P2):}

Criado em 1988, e revitalizado em 1995, é uma ampla área de lazer, das mais procuradas pela população local. Conhecido também como lago municipal de Cascavel, o parque é composto por áreas florestadas e um lago artificial, criado pelo represamento do rio Cascavel e córregos de pequeno porte. O parque abrange 93,35 ha, sendo 55,35 ha de mata e 38 ha de lâmina d'água. Está localizado em área urbana do município de Cascavel $\left(24^{\circ} 57^{\prime} 41.32\right.$ 'S e $56^{\circ}$ 26'14.62'O) (TAVARES; VALENTE-MOREIRA, 2000) (Figura 1). É composto de vegetação caracterizado como floresta ombrófila mista, embora em muitos pontos tenha sido realizado plantio de espécies exóticas. 
Figura 1: Imagem de satélite dos Parques Tarquínio Joslin dos Santos (P1) e Parque Ecológico Paulo Gorski (P2). (Fonte Google Earth, 2014).

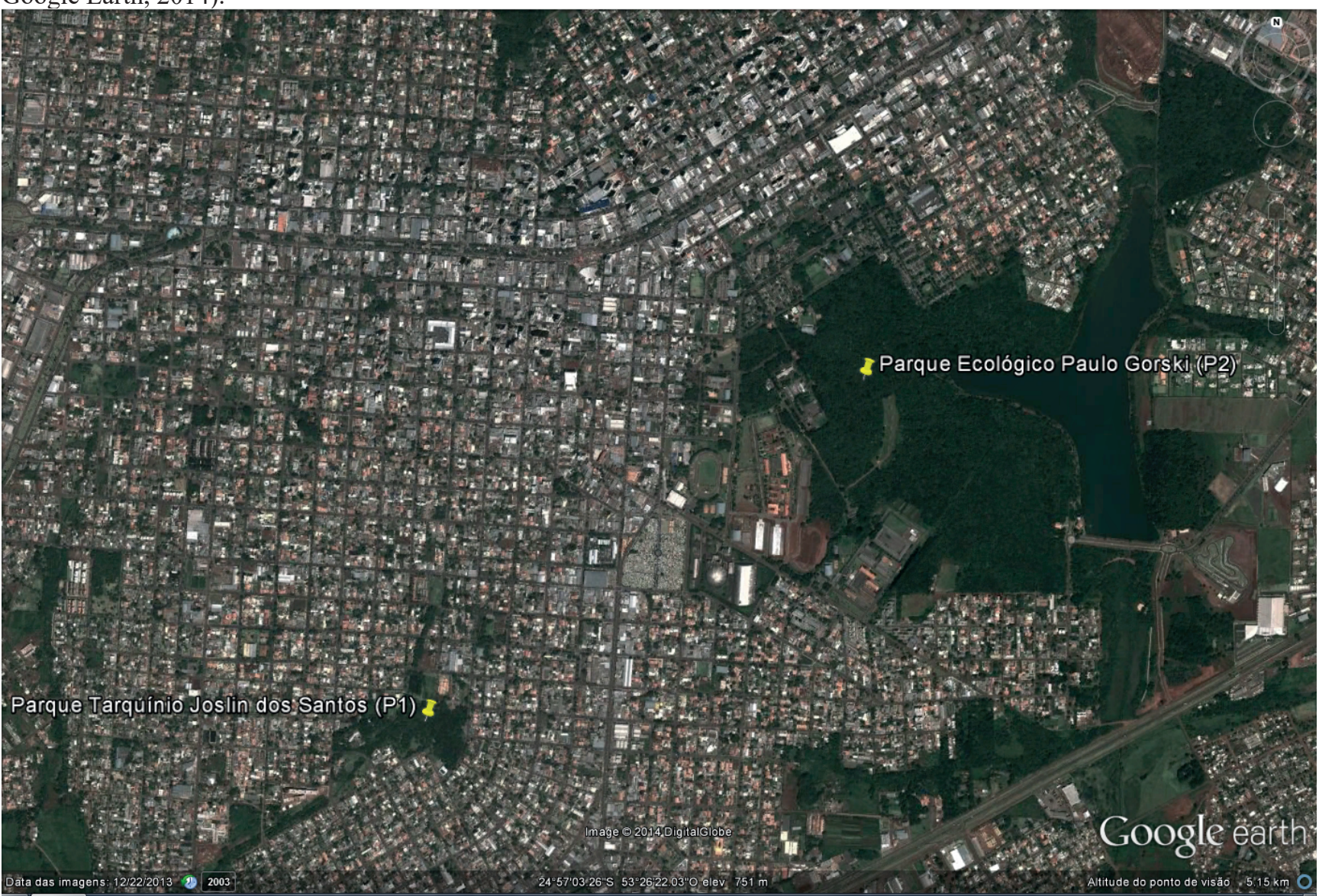

\section{Coleta de Dados}

O estudo foi realizado entre dezembro de 2007 a outubro de 2008, nos parques P1 e P2. O levantamento em campo foi realizado semanalmente, nos períodos matutino e vespertino.

O levantamento qualitativo das espécies de aves foi realizado por observações diretas com auxílio de binóculo, máquina fotográfica e guia de identificação de aves (NAROSKY; YZURIETA, 2003).

O comportamento reprodutivo foi analisado por meio da avaliação da morfometria dos ninhos e estratégias reprodutivas. Os ninhos ativos encontrados foram acompanhados durante o período de ocupação, para descrição do comportamento reprodutivo da espécie.

A obtenção dos dados morfométricos dos ninhos foi realizada mediante medições, da profundidade da câmara oológica, altura dos ninhos em relação ao solo, comprimento do topo até a base, diâmetro maior e menor, descrição da forma, substrato de apoio e material para a construção.

As estratégias de reprodução foram analisadas estimando-se o tamanho da espécie vegetal utilizada para nidificação; quantidade, coloração e forma dos ovos e cuidado parental. $\mathrm{Na}$ análise do cuidado parental foi determinado, quando possível, o tempo de permanência dos pais no ninho alimentando os filhotes e/ou comportamentos de vigília e defesa do ninho, incluindo o número de vezes que o comportamento for executado pelos mesmos durante a observação.

\section{Resultados e Discussão}

\section{Composição avifaunística}

A tabela 01 descreve a composição avifaunística de P1 e P2, totalizando 58 espécies pertencentes à 28 famílias, representadas por 13 ordens, sendo Tyrannidae, a família mais representativa em ambos os parques, com oito e seis espécies, respectivamente. Em P1 foram registradas a presença de 38 espécies pertencentes à 20 famílias, representada por oito ordens, já em $\mathrm{P} 2$ foram registradas a presença de 38 espécies pertencentes à 21 famílias e 12 ordens, com destaque também a família Columbidae que representou cinco espécies. Segundo Marques e Pachaly (2010) o número de espécies de aves é proporcional à área, além do grau de antropização. As áreas estudadas recebem influência humana, sendo utilizados como área de lazer, assim a perda de hábitat e a fragmentação da parte verde podem ter influenciado a estrutura das comunidades de aves, o que está diretamente relacionado com o número de espécies encontradas nos parques. 
Tabela 1: Lista das espécies encontradas nos Parques Tarquínio dos Santos (P1) e Ecológico Paulo Gorski (P2).

\begin{tabular}{|c|c|c|c|c|}
\hline ORDEM & FAMÍLIA & ESPÉCIE & NOME POPULAR & LOCAL \\
\hline \multirow{3}{*}{ Anseriformes } & \multirow{3}{*}{ Anotidae } & Anser anser & Ganso doméstico & $\mathrm{P} 2$ \\
\hline & & Anas versicolor (Vieillot, 1816) & Marreca cri-cri & $\mathrm{P} 2$ \\
\hline & & Cairina moschata (Linnaeus, 1758) & Pato-do-mato & $\mathrm{P} 2$ \\
\hline \multirow[t]{2}{*}{ Apodiformes } & \multirow[t]{2}{*}{ Trochilidae } & $\begin{array}{l}\text { Anthracothorax nigricollis (Vieillot, } \\
\text { 1817) }\end{array}$ & Beija-flor-de-veste-preta & $\mathrm{P} 1$ \\
\hline & & Chlorostilbon lucidus (Shaw, 1812) & Besourinho-de-bico-vemelho & $\mathrm{P} 1$ \\
\hline Cathartiformes & Cathartidae & Coragyps atratus (Bechstein, 1793) & Urubu-de-cabeça-preta & $\mathrm{P} 1 / \mathrm{P} 2$ \\
\hline \multirow[t]{2}{*}{ Charadriiformes } & Charadriidae & Vanellus chilensis (Molina, 1782) & Quero-quero & $\mathrm{P} 1 / \mathrm{P} 2$ \\
\hline & Jacanidae & Jacana jacana (Linnaeus, 1766) & Jaçanã & P2 \\
\hline Ciconiiformes & Ardeidae & Butorides striata (Linnaeus, 1758) & Socozinho & $\mathrm{P} 2$ \\
\hline \multirow{5}{*}{ Columbiformes } & \multirow{5}{*}{ Columbidae } & Columba livia (Gmelin, 1789) & Pombo-doméstico & $\mathrm{P} 2$ \\
\hline & & Columbina talpacoti (Temminck, 1811) & Rolinha-roxa & $\mathrm{P} 1 / \mathrm{P} 2$ \\
\hline & & Leptotila verreauxi (Bonaparte, 1855) & Juriti-pupu & P2 \\
\hline & & $\begin{array}{l}\text { Patagioenas picazuro (Temminck, } \\
1813)\end{array}$ & Pombão & $\mathrm{P} 2$ \\
\hline & & Zenaida auriculata (Des Murs, 1847) & Pomba-de-bando & $\mathrm{P} 1 / \mathrm{P} 2$ \\
\hline Coraciiformes & Alcedinidae & Megaceryle torquata (Linnaeus, 1766 & Martim-pescador-grande & P2 \\
\hline \multirow{3}{*}{ Cuculiformes } & \multirow{3}{*}{ Cuculidae } & Crotophaga ani Linnaeus, 1758) & Anu preto & $\mathrm{P} 1 / \mathrm{P} 2$ \\
\hline & & Guira guira (Gmelin, 1788) & Anu branco & $\mathrm{P} 1 / \mathrm{P} 2$ \\
\hline & & Piaya cayana (Linnaeus, 1766) & Alma-de-gato & $\mathrm{P} 1 / \mathrm{P} 2$ \\
\hline \multirow{2}{*}{ Falconiformes } & Accipitridae & Rupornis magnirostris (Gmelin, 1788) & Gavião-carijó & $\mathrm{P} 1$ \\
\hline & Falconidae & Caracara plancus (Miller, 1777) & Caracará & $\mathrm{P} 1 / \mathrm{P} 2$ \\
\hline Gruiformes & Rallidae & Gallinula chloropus (Linnaeus, 1758) & Frango-d'água-comum & P2 \\
\hline \multirow{22}{*}{ Passeriformes } & \multirow{5}{*}{ Emberizidae } & Sicalis flaveola (Linnaeus, 1766) & Canário-da-terra-verdadeiro & $\mathrm{P} 1$ \\
\hline & & Sporophila lineola (Linnaeus, 1758) & Bigodinho & $\mathrm{P} 2$ \\
\hline & & Sporophila plumbea (Wied, 1830) & Patativa & $\mathrm{P} 1$ \\
\hline & & Volatinia jacarina (Linnaeus, 1766) & Tizio & $\mathrm{P} 1$ \\
\hline & & $\begin{array}{l}\text { Zonotrichia capensis (Statius Muller, } \\
1776 \text { ) }\end{array}$ & Tico-tico & $\mathrm{P} 2$ \\
\hline & Estrildidae & Estrilda astrild (Linnaeus, 1758) & Bico-de-lacre & $\mathrm{P} 1$ \\
\hline & Fringillidae & Euphonia chlorotica (Linnaeus, 1766) & Fim-fim & $\mathrm{P} 1$ \\
\hline & Furnariidae & Furnarius rufus (Gmelin, 1788) & João-de-barro & $\mathrm{P} 1 / \mathrm{P} 2$ \\
\hline & \multirow[t]{2}{*}{ Hirundinidae } & $\begin{array}{l}\text { Pygochelidon cyanoleuca (Vieillot, } \\
1817 \text { ) }\end{array}$ & Andorinha-pequena-de-casa & $\mathrm{P} 1$ \\
\hline & & Riparia riparia (Linnaeus, 1758) & Andorinha-do-barranco & $\mathrm{P} 1$ \\
\hline & \multirow{3}{*}{ Icteridae } & Gnorimopsar chopi (Vieillot, 1819) & Graúna & $\mathrm{P} 1$ \\
\hline & & Molothrus bonariensis (Gmelin, 1789) & Vira-bosta & $\mathrm{P} 2$ \\
\hline & & Molothrus rufoaxillaris (Cassin, 1866) & Vira-bosta-picumã & P1 \\
\hline & Mimidae & Mimus saturninus (Lichtenstein, 1823) & Sabiá-do-campo & $\mathrm{P} 2$ \\
\hline & Motacillidae & Anthus lutescens (Pucheran, 1855) & Caminheiro-zumbidor & $\mathrm{P} 1$ \\
\hline & \multirow{2}{*}{ Parulidae } & Basileuterus flaveolus (Baird, 1865) & Canário-do-mato & $\mathrm{P} 1$ \\
\hline & & Dendroica striata (Forster, 1772) & Mariquita-de-perna-clara & $\mathrm{P} 1$ \\
\hline & Passeridae & Passer domesticus (Linnaeus, 1758) & Pardal & $\mathrm{P} 1 / \mathrm{P} 2$ \\
\hline & Thamnophilidae & $\begin{array}{l}\text { Thamnophilus caerulescens Vieillot, } \\
1816\end{array}$ & Choca-da-mata & $\mathrm{P} 2$ \\
\hline & \multirow{2}{*}{ Thraupidae } & Tersina viridis (Illiger, 1811) & Saí-andorinha & $\mathrm{P} 1 / \mathrm{P} 2$ \\
\hline & & Thraupis sayaca (Linnaeus, 1766) & Sonhaçu-cinzento & $\mathrm{P} 1 / \mathrm{P} 2$ \\
\hline & Turdidae & Turdus amaurochalinus (Cabanis, 1850) & Sabiá-poca & P2 \\
\hline
\end{tabular}




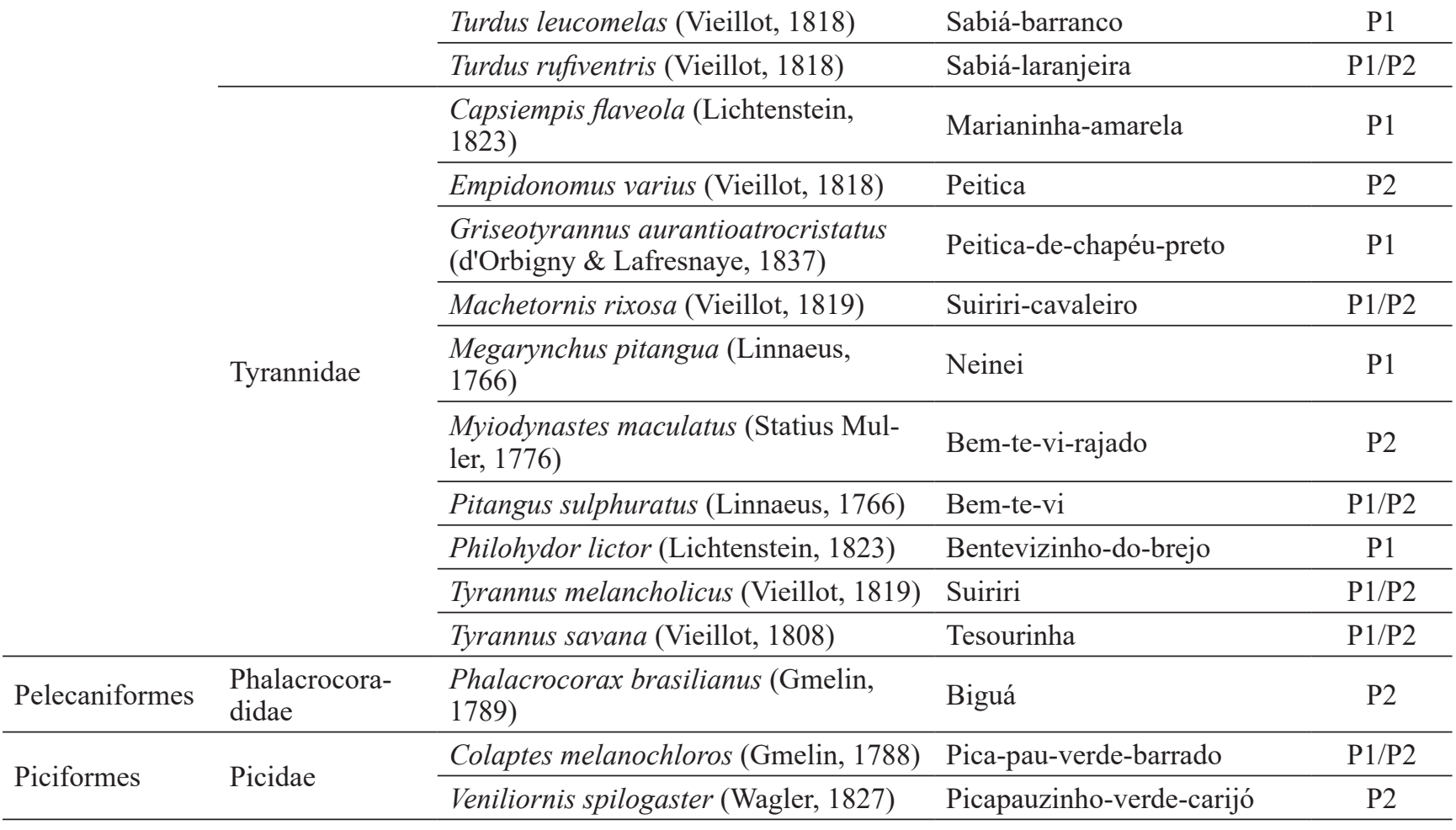

É importante destacar em P1 a presença de Basileuterus flaveolus e Sporophila plumbea, por se tratarem de espécies vulneráveis que correm risco de extinção. Segundo o Instituto Ambiental do Paraná (2007), Basileuterus flaveolus é encontrada apenas no norte do estado do Paraná, onde se encontra restrita a alguns poucos e pequenos fragmentos de Floresta Estacional Semidecidual, fato este que corrobora o encontrado neste trabalho, onde a espécie foi observada no oeste do estado. Não bastasse a sua pequena e esporádica distribuição, a espécie é intensamente ameaçada por desastres naturais e fatores antrópicos, já que esta é uma espécie dependente de micro-habitat ligado a cursos d'água. O Instituto Ambiental do Paraná (2007) destaca ainda, a necessidade de medidas efetivas de conservação que precisam ser tomadas localmente, e a importância de se estabelecerem Unidades de Conservação para esta espécie. Já Sporophila plumbea é uma espécie muito capturada para manutenção em cativeiro e comércio ilegal, o que tem diminuído intensamente o número de indivíduos da espécie, além disso, ela apresenta uma distribuição regional restrita, ocorrendo em paisagens abertas, como os Campos Naturais e Cerrado. Além dos fatores descritos anteriormente, a Sporophila plumbea ainda é ameaçada pela constante perda de hábitat, onde acaba perdendo seus sítios de nidificação e alimentação. Devido à pequena extensão paranaense desses hábitats ocupados pela espécie, a necessidade de conservação da espécie se faz em nível de urgência, sugerindo-se ainda, estudos que vinculem a biologia da espécie para que, se assim for necessário, possa-se efetuar um manejo tanto da espécie quanto do hábitat ocupado pela mesma.

Nos meses de abril e de maio de 2008, observou-se a presença de Dendroica estriata, que é uma espécie migratória da América do Norte, alimentando-se no P1. Esta espécie possui hábitos arbóreos e ocupa hábitats florestais, embora durante a migração, é vista alimentando-se no chão. É registrada frequentemente na Venezuela, onde ocorre como ave de passagem, sendo ocasionalmente visualizada nas Guianas, Colômbia, Equador, Peru e na Amazônia Brasileira, além de ser registrada no Rio de Janeiro e São Paulo (SICK, 1997), não havendo nenhum registro no Paraná.

Poletto (2002) trabalhou com levantamento de aves em P2, registrando a ocorrência de 77 espécies pertencentes à 15 ordens e 34 famílias. A fim de confirmar a presença destas espécies, o levantamento foi novamente efetuado, pois ele exerce profunda relação com a nidificação também estudada no Parque. $\mathrm{O}$ fato de terem sido registradas 38 espécies pertencentes à 21 famílias e 12 ordens neste estudo é algo a ser investigado, visto que inúmeros fatores podem colaborar para tal situação. P2 é uma área muito antropizada, pessoas circulam pelos arredores, e muitas vezes a ação causada pela população não é vantajosa, a presença da população causa muito barulho e pode prejudicar a permanência das espécies no local, causando a fragmentação de habitat e perda da parte verde. Possivelmente tal situação foi provocada em função das condições climáticas que se alteram de um ano para outro, como precipitação, umidade, temperaturas mínima/média/máxima.

Dentre as espécies observadas em P2, nenhuma se encontra em riscos de extinção (IAP, 2006).

\section{Caracterização do comportamento reprodutivo}

\subsection{Parque Tarquínio Joslin dos Santos (P1)}

O comportamento reprodutivo foi analisado de fevereiro a setembro de 2008. As nidificações de Zenaida auriculata, Furnarius rufus, Pitangus sulphuratus e Machetornis rixosa foram registradas no parque. Em função de dificuldade de acesso foi realizado a morfometria dos ninhos apenas para Zenaida auriculata. Os ninhos (oito) se apresentaram na forma de plataforma, como diagnosticado no trabalho de Guaraldo e Gussoni (2006), sendo que as médias morfomé- 
tricas foram: 1,90 m de altura em relação ao solo; $5,75 \mathrm{~cm}$ de topolbase; $0,75 \mathrm{~cm}$ profundidade; $13,62 \mathrm{~cm}$ diâmetro maior e $10,62 \mathrm{~cm}$ diâmetro menor; em relação ao substrato de apoio, predominam uma forquilha entre dois galhos, tronco seco e apenas um galho como suporte. O material para construção foi o parâmetro que mais variou, visto que os ninhos apresentavam, em sua grande maioria, gavinhas e gravetos, mas ainda foram encontrados outros materiais como folhas, raízes, fios de nylon e frutos secos, como verificados no trabalho de Guaraldo e Gussoni (2006). Zenaida auriculata é uma espécie que não apresenta especialidades em construção de seus ninhos, assim sua estratégia de reprodução, durante a nidificação, é permanecer todo tempo sobre o ninho ou próximo dele.

Os ninhos apresentaram dois ovos, com coloração branca, também observado por Guaraldo e Gussoni (2006). $\mathrm{Na}$ fase de incubação dos ovos foi verificada a presença de um individuo adulto sobre o ninho. $\mathrm{O}$ individuo adulto não saía do ninho, nem mesmo com a aproximação do coletor, isto pode estar relacionado à falta de camuflagem dos ovos e os ninhos ralos construídos pela espécie. Sick (1997) afirma que esse comportamento está diretamente relacionado com o cuidado parental, pois o fato do individuo permanecer sobre o ninho, parece, para o predador, que ele está ferido, assim despistaria uma ameaça. Em um dos ninhos, foi possível verificar a eclosão de apenas um dos ovos. É imprescindível ressaltar, que o acompanhamento do desenvolvimento dos ninhegos, foi interrompido devido ao vandalismo. $\mathrm{O}$ fato de Zenaida auriculata ser abundante em relação à nidificação em P1 pode estar ligada a fácil adaptação que as avoantes apresentam em ambientes modificados. Sick(1997) menciona que a espécie modifica seus hábitos naturais para se adaptar a novas alterações ambientais.

O fato de ocorrerem 38 espécies e apenas quarto destas nidificarem em P1 é algo a ser investigado, visto que inúmeros fatores colaboram para essa situação. O Parque é pequeno e uma área bastante antropizada, pessoas circulam pelos arredores, e muitas vezes a ação causada pela população não é vantajosa, já que, como registrado no presente trabalho, um ninho foi destruído devido ao vandalismo, além disso, a presença da população causa muito barulho e prejudica a permanência das espécies no local. Ainda, existem espécies de aves que nidificam em substratos específicos, e P1 é pouco diversificado floristicamente, inclusive foram introduzidas algumas espécies vegetais no local, o que poderia estar afetando a nidificação de determinadas espécies. Algumas espécies podem nidificar nos meses em que não foi realizado o estudo (outubro a janeiro). Algumas espécies podem estar usando a área apenas como pontos de alimentação, repouso e ainda serem migrantes, como foi o caso de Dendroica srtiata. Além disso, outro fato que contribui para o baixo número de espécies nidificantes em P1 é a camuflagem de ninhos, a inacessibilidade e demais estratégias de reprodução de algumas espécies.

\section{Parque Ecológico Paulo Gorski (P2)}

O comportamento reprodutivo foi analisado de dezembro de 2007 à outubro de 2008. As espécies encontradas nidificando no período foram Zenaida auriculata (16 ninhos), Furnarius rufus (seis ninhos), Machetornis rixosa (um ninho), Megarhynchus pitanguá (um ninho), Turdus rufiventris (um ninho), Crotophaga ani (um ninho) e Butorides striata (um ninho). Dentre dos ninhos que passaram pela fase de morfometria, dos identificados, apenas o ninho de Crotophaga ani, e 11 dos ninhos de Zenaida auriculata, puderam ser descritos, os demais devido a altura em que se encontravam, impossibilitaram a coleta.

Em dezembro foi registrado um ninho ativo de Crotophaga ani (anu preto), este se apresentava alargado e arredondado, apoiado em uma forquilha com dois galhos, possuía $20 \mathrm{~cm}$ de topo \base; oito centímetros de profundidade da câmara oológica; $25 \mathrm{~cm}$ de diâmetro maior; $20 \mathrm{~cm}$ de diâmetro menor e 2,30 m de altura em relação ao solo. Além disso, o ninho foi construído com pequenos galhos, gravetos e gavinhas, nele foram encontrados sete ovos elípticos, azul-esverdeados. Sick (1997), afirma que ninhos de Crotophaga ani são grandes, profundos e os ovos são azul-esverdeados, podendo variar de quatro a sete por ninho, estes recebem cuidados de até 20 indivíduos (nidificação é coletiva). Observou-se a constante presença de um indivíduo adulto em uma árvore próxima ao ninho que vocalizava intensamente em alta frequência, conforme o coletor se aproximava do ninho, maior era a vocalização e mais próximo o adulto chegava do coletor, três minutos após a vocalização apareceram mais quatro indivíduos, que vocalizavam intensamente, e então começaram a sobrevoar o local até o término da coleta. Um fato interessante em relação à nidificação da espécie é a presença de folhas verdes no interior do ninho, como verificado por Sick (1997), o qual descreve que este comportamento é efetuado pelos indivíduos que chocam o ninho, como forma de camuflagem.

A Zenaida auriculata apresenta ninhos na forma plataforma, onde as médias dos dados morfométricos são: altura em relação ao solo $2,22 \mathrm{~m}$; topo $\backslash$ base $4,45 \mathrm{~cm}$; profundidade da câmara oológica 1,09 cm; diâmetro maior $9,90 \mathrm{~cm}$ e diâmetro menor 7,0 cm. Em relação ao substrato de apoio predominaram forquilha entre dois galhos, apenas um galho, onde geralmente, os ninhos se encontravam mais na extremidade, sobre vários galhos finos e um deles estava na cavidade de um tronco seco. Quanto ao material de construção, foram observadas a presença de folhas, gavinhas, gravetos e raízes, dados estes que confirmam os verificados no trabalho de Guaraldo e Gussoni (2006).

Cinco ninhos de Zenaida auriculata, estavam ativos, destes foi possível verificar a construção ou reforma de um ninho em um Syagrus romanzoffiana (Cham.) Glassman (Jerivá), onde um indivíduo permanecia sobre o ninho e o outro procurava o material de construção no solo, como verificado por Guaraldo e Gussoni (2006). Observou-se dois ninhos em período de incubação, nestes havia dois ovos brancos cada, como afirmado por Sick (1997) e Guaraldo e Gussoni (2006). Verificou-se também a incubação de dois ninhos contendo dois ninhegos, em um desses ninhos o adulto incubou apenas um dos filhotes. Um comportamento registrado e interessante foi a presença de um filhote de tamanho médio, sobre um galho que recebeu cuidado do casal, enquanto um o alimentava com sementes, o outro atacava indivíduos que se aproximassem do local. Sick (1997), afirma que o casal alimenta os filhotes com sementes depois que estes saem do ninho.

A nidificação da espécie Turdus rulfiventris foi verificada pela presença de um ninho que se encontrava a apro- 
ximadamente seis metros de altura em relação ao solo, o que impossibilitou a morfometria. Observou-se a incubação de um ou mais filhotes, assim identificada pela vocalização do mesmo(s). Com a aproximação do coletor à árvore em que se encontrava o ninho, o adulto em prol de defesa, executou vôos rasantes e próximos ao observador. Além disso, foi registrada a alimentação, na qual o adulto alimentava o filhote a cada oito minutos, sendo que o adulto permanecia no ninho após a alimentação por três minutos. Segundo Almeida, Evangelista e Silva (2012), a alimentação normalmente é feita pelo casal e mesmo após a saída do ninho como estratégia de evitar a predação neste período.

Furnarius rufus apresentou seis ninhos ativos, encontrando-se em locais inacessíveis, sempre na extremidade dos galhos, em forma de forno e construídos com barro, como verificado no trabalho de Pereira et al. (2006). Foi possível observar que o cuidado parental é feito pelo casal, alternadamente. Na alimentação, enquanto um indivíduo busca alimento o outro ficava no ninho, assim que aquele chegava com o alimento, vocalizava intensamente e mantinha uma postura ereta na entrada no ninho, então o outro adulto saía do ninho para que este entrasse e alimentasse o filhote. Este comportamento alternado se repetiu por duas vezes, depois das duas aves permaneceram dentro do ninho.

A nidificação de Machetornis rixosa, Megarhynchus pitangua foi verificada pela presença de incubação de um indivíduo adulto sobre o ninho e Butorides striata pela presença do adulto com dois filhotes.

$\mathrm{O}$ fato das demais espécies não nidificarem em P2 pode estar relacionada aos mesmos fatores citados para o P1

\section{Conclusão}

O Parque Tarquínio Joslin dos Santos e o Parque Ecológico Paulo Gorski apresentam composição avifaunística relevante, e algumas espécies ocupam estes locais como sítios de nidificação, repouso e alimentação. Vale ressaltar também que foram encontradas duas espécies em risco de extinção (Basileuterus flaveolus, Sporophila plúmbea) e necessitam ser preservadas, assim como os locais que elas ocorrem, sendo imprescindível destacar a importância da manutenção de Parques em ambiente urbano.

Os parques urbanos sofrem muita influência antrópica, e grande parte da população que ocupa estas áreas como lazer, acaba interferindo na nidificação das espécies, talvez por falta de incentivo e conhecimento sobre o assunto, assim pode-se sugerir que mais trabalhos em âmbito ambiental e educacional, para que as espécies encontradas possam permanecer nos parques e assim também nidificarem.

\section{Referências}

ALMEIDA, M. S.; EVANGELISTA, M. M.; SILVA, A. J. E. Biologia da nidificação de aves no município de Porto Esperidião, Mato Grosso. Atualidades ornitológicas, n. 167 , p. 51-56, 2012.

BARTHOLOMEW, G. A. The role of natural history in contemporary biology. BioScience, v. 36, n. 5, p. 324-329, 1986.
CONTERNO, S.; CÂNDIDO JUNIOR, J. F. Levantamento preliminar da avifauna e estudo de sua distribuição dentro das diferentes fisionomias vegetativas do Parque Ambiental de Cascavel, em Cascavel - PR. In: SEMANA DA BIOLOGIA, 11., 2001, Cascavel. Universidade Estadual do Oeste do Paraná. Disponível em: <http://www.unioeste.br/ prg/download/XI_Semana_de_Biologia2001.pdf $>$. Acesso em: 25 jun. 2014.

COSTA, L. C. M. O Comportamento Interespecífico de Defesa do Quero-quero, Vanellus chilensis (Molina, 1782) (Charadriiformes, Charadriidae). Revista de Etologia, v. 4, n. 2, p. 95-108, 2002.

\section{DE CARVALHO, A. O uso de ambiente urbano por} Falconiformes, tendo como ênfase o Quiriquiri Falco Sparverius Linnaeus, 1758 (Falconidae, falconiformes). 2006. 63f. Monografia (Trabalho de Conclusão de Curso de Ciências Biológicas) - Universidade Estadual do Oeste do Paraná, Cascavel, 2006.

GUARALDO, A. C.; GUSSONI, C. O. A. Nidificação de Zenaida auriculata (Des Murs, 1847) no município de rio claro, estado de São Paulo, sudeste do Brasil. In: CONGRESSO BRASILEIRO DE ORNITOLOGIA, 14., 2006, Ouro Preto. Anais... Ouro Preto: Sociedade Brasileira de Ornitologia, 2006. p. 14.

HATCHWELL, B. J. et al. Reproductive success and nestsite selection in a cooperative breeder: Effect of experience and a direct benefit of helping. The Auk, v. 116, n. 2, p. 355-363, abr 1999.

\section{INSTITUTO AMBIENTAL DO PARANÁ. Fauna do Paraná em Extinção. Curitiba, 2007. 272 p.}

LARISON, B. et al. Song Sparrows Vs. cowbird brood parasites: Impacts of forest structure and nest-site selection. The Condor, v. 100, n. 1, p. 93-101, 1998.

LOPES, E. V.; ANJOS, L. A composição da avifauna do campus da Universidade Estadual de Londrina, norte do Paraná, Brasil. Revista Brasileira de Zoologia, v. 23, n. 1, p.145-146, 2006.

LOPES, E. V.; ANJOS, L. Observações sobre a reprodução de Nyctibius griseus no campus da Universidade Estadual de Londrina, norte do Paraná. Ararajuba, v. 13, n. 1, p. 109-112, 2005.

LOURES-RIBEIRO, A.; GIMENES, M. R.; ANJOS, L. Observações sobre o comportamento reprodutivo de Ictinia plumbea (Falconiformes, Accipitridae) no campus da Universidade Estadual de Maringá, Paraná, Brasil. Ararajuba, v. 11, n. 1, p. 85-87, 2003.

MARQUES, R. A.; PACHALY, J. R. Levantamento da ornitofauna da trilha ecológica Peroba-Rosa, no município de Altônia, Paraná. Arquivos de Ciências Veterinárias e Zoologia da UNIPAR, Umuarama, v. 13, n. 1, p. 9-14, 2010 . 


\section{NAROSKY, T.; YZURIETA, D. Guía para la}

identificación de las aves de Argentina y Uruguay.

Argentina: Vazquez Mazzini Editores, 2003. 345 p.

OLMOS, F. Nest location, clutch size and nest success in the Scarlet Ibis Eudocimus ruber. International Journal of Avian Science, v. 145, n. 1, p. 12-18, 2003.

OPPLIGER, A.; RICHNER, H.; CHRISTE, P. Effects of an ectoparasite on lay date, nest-site choice, desertion, and hatching success in the great tit (Parus major). Behavioral Ecology. v. 5, n. 2, p. 130-134, 1994.

PEREIRA, Z. P. et al. Monitoramento de ninhos de Furnarius rufus no Parque do Sabiá/Uberlândia/Mg. In: CONGRESSO BRASILEIRO DE ORNITOLOGIA, 14., 2006, Ouro Preto. Anais... Ouro Preto: Sociedade Brasileira de Ornitologia, 2006. p. 11.

POLETTO, F. Monitoramento de aves paludícolas do Lago Municipal de Cascavel Paraná e levantamento avifaunístico. 2002. 50 f. Trabalho de Conclusão de Curso (Monografia) - Universidade Estadual do Oeste do Paraná UNIOESTE, Cascavel - Paraná, 2002

PREFEITURA MUNICIPAL DE CASCAVEL. Disponível em: $<$ http://www.cascavel.pr.gov.br/>. Acesso em: 15 mar. 2008.

ROBINSON, T. R.; ROBINSON, W. D.; EDWARDS, E. C. Breeding ecology and nest-site selection of songs wrens in Central Panama. The Auk, v. 117, n. 2, p. 345-354, 2000.

RODRIGUES, M. Parental care and polygyny in the chiffchaff Phylloscopus collybita, Behaviour. v. 133, n. 13/14, p. 1077-1094, 1996.

SCHERER, J. F. M. et al. Estudo da avifauna associada à área úmida situada no Parque Mascarenhas de Moraes, zona urbana de Porto Alegre (RS). Biotemas, v. 19, n. 1, p. 107110, 2006.

SICK, H. Ornitologia Brasileira. Rio de Janeiro: Nova Fronteira, 1997. 912 p.

SMITH, K. W. Nest site selection of the great spotted woodpecker Dendrocopus major in two oak woods in southern England and its implications for woodland management. Biological Conservation. v. 80, n. 3, p.283288, 1997.

TAVARES, B.; VALENTE-MOREIRA, I. M.

Diatomoflórula do lago de Cascavel, município de Cascavel, estado do Paraná, Brasil. Hoehnea, v. 27, n. 1, p. $1-24,2000$. 